\title{
TRACER: a toolkit to register and visualize anatomical coordinates in the rat brain
}

Jacopo Paglia ${ }^{1 \dagger \sharp}$, Pearl Saldanha ${ }^{1 \dagger^{*}}$, Jingyi Guo Fuglstad ${ }^{1 \ddagger}$, Jonathan R. Whitlock $^{1 \ddagger}$

1 Kavli Institute for Systems Neuroscience, Norwegian University of Science and Technology

(NTNU), Olav Kyrres Gate 9, 7089 Trondheim, Norway

Present address: ${ }^{\sharp}$ KnowBe4, Apotekergata 10, Oslo, Norway

$\dagger$ These authors contributed equally to this work; ${ }^{\ddagger}$ These authors jointly supervised this work

*Correspondence: pearl.saldanhda@ntnu.no; jonathan.whitlock@ntnu.no

\begin{abstract}
The need for anatomical registration and visualization tools is greater than ever thanks to novel technologies that allow users to record from thousands of neurons across multiple brain regions simultaneously. The vast majority of digital reconstruction toolkits for rodent models were developed using mouse brain atlases, leaving few options for those using rats. Retrofitting rat atlases into extant software is possible, but this demands effort and programming skills which most end-users lack. We therefore developed an open-source, python-based Toolkit for Reconstructing Anatomical CoordinatEs in Rats, TRACER, which allows users to reconstruct the trajectories of recording electrodes (e.g Neuropixels) or to visualize virus expression or other features in a comprehensively annotated 3D volume, the Waxholm Space (WHS) rat brain atlas. The WHS atlas is made from high-resolution magnetic resonance imaging (MRI) and diffusion tensor imaging (DTI) scans, and contains $>200$ regional delineations. Beyond post-hoc reconstructions of electrodes or virus expression, TRACER can be used to generate coordinates for targeting brain regions prior to surgery. TRACER is available openly on GitHub and is compatible with Windows, macOS and Linux operating systems.
\end{abstract}

\section{Introduction}

Any physiological or anatomical study of the brain requires that one can target regions of interest accurately prior to experiments and, afterward, generate an objective, accessible record of the areas studied. The procedure of registering histological data in a reference atlas, though critical, is usually time consuming and difficult, which has prompted the development of digital toolkits to accelerate and systematize the process. In the rodent field, and for mice in particular, several visualization toolkits (e.g Shamash et al. 2018; Song et al. 2020; Claudi et al. 2021) and reference atlases (e.g. George Paxinos 2008; Wang et al. 2020) are available, which together give users the ability to reconstruct electrode tracks, viral expression, anatomical projections (Oh et al. 2014; Zingg et al. 2014; Winnubst et al. 2019), cell types (Fürth et al. 2018), gene expression patterns or functional nodes (Lein et al. 2007; Ortiz et al. 2020). Although annotated 3D atlases have been produced for rats (e.g. Toga et al. 1995; Hjornevik et al. 2007; Calabrese et al. 2013; Papp et al. 2014;Papp et al. 2015;), few, if any, open source toolkits incorporate them, leaving most rat users to still perform reconstructions manually. To address this methodological gap we developed TRACER, a toolkit specifically for rat users, in which regions of interest are marked on standard 2D histological images and loaded into a comprehensively annotated 3D volume, the WHS atlas of the Sprague Dawley rat brain (Papp et al. 2014, v4). The WHS atlas consists of two atlas elements: a reference data set 
made from micron-resolution, isotropic MRI and DTI scans of an adult male Sprague Dawley rat brain, and delineations of over 200 brain regions defined in the MRI reference data set.

TRACER was developed initially to reconstruct the trajectories of silicon probes, such as Neuropixels, and to identify which electrode sites traversed which brain regions during in vivo recordings. It can reconstruct electrode tracks for up to 6 probes within one animal or across multiple animals and, similar to previous mouse brain toolkits (Shamash et al. 2018), it can calculate anatomical coordinates and insertion angles to target desired brain regions when planning a surgery. Beyond electrophysiology, TRACER can be used to visualize 3D volumes of virus expression, anatomical tracers or pathological degeneration extending through multiple sections, and it works equally well in coronal, horizontal and sagittal planes. TRACER is also compatible with potentially any method of tissue staining, so long as the regions of interest are visible and can be delineated by the user. Detailed annotations are provided automatically once a region of interest (ROI) has been reconstructed in the WHS 3D volume. The toolkit was written as a downloadable python package to maximize general accessibility, and the software is compatible with Windows, macOS and Linux users. TRACER is intended as an open source community resource that is available for further expansion and refinement and can be downloaded from. We hope it offers a useful addition to the anatomical methods available for rat users and the neuroscience community at large.

\section{Materials and Methods}

\section{Python packages and resources}

The general outline of TRACER is written entirely in python version three using basic python packages such as numpy, matplotlib, math. The package Vedo was used for $3 \mathrm{D}$ visualizations of the brain. More extensive detail specifying the versions of packages used in TRACER can be found in the requirements.txt file. Documentation and step-wise instructions for installing and implementing TRACER can be found in the user manual that comes with the software. All of TRACER's code is open-source and is available at the Github repository. To use TRACER we recommend users to create an Anaconda virtual environment with python (Version 3.8.5) and other supporting packages which are specified in the requirements text file in the TRACER package.

Table 1. Key resources for TRACER

\begin{tabular}{lll}
\hline Resource & Type & Source and reference \\
\hline Software, algorithm & Python 3.8.5 & Van Rossum and Drake 2009 \\
Software, algorithm & Numpy & Harris et al. 2020 \\
Software, algorithm & Scipy & Virtanen et al. 2020 \\
Software, algorithm & Vedo & https://doi.org/10.5281/zenodo.4287635 \\
Software, algorithm & Matplotlib & Hunter 2007 \\
Software, algorithm & Scikit-image & Walt et al. 2014 \\
\hline
\end{tabular}

\section{Neuropixels, MRI scanning and virus expression}

All experiments were performed in accordance with the Norwegian Animal Welfare Act and the European Convention for the Protection of Vertebrate Animals used for Experimental and Other Scientific Purposes. All experiments were approved by the Norwegian Food Safety Authority (Mattilsynet; protocol IDs 27175 and 25094). All tissue for in-house testing came from adult (>15wk) Long-Evans hooded rats. Detailed steps of the surgical preparation and post-operative care are described in Mimica et al. 2018. 
Neuropixels: the probes (version 1.0 Neuropixels, IMEC, Belgium) were coated with DiI (Vybrant DiI, catalog no.V22888, Thermo Fisher Scientific, USA) by repeatedly drawing a $2 \mu \mathrm{L}$ droplet of DiI solution at the tip of a micropipette up and down the length of the probe shank until all DiI had dried onto the shank, causing the shank to appear pink. The probes were angled appropriately in the arm of a stereotaxic frame and inserted at a rate of $100-300 \mu \mathrm{m}$ per minute. Chronically implanted animals were kept $<1$ week following surgery, after which they were perfused with $4 \%$ paraformaldehyde and the brains were removed. The shanks of the Neuropixels were snapped and remained in the brains to give enhanced contrast and visibility during subsequent MRI acquisition.

Following MRI scanning, the brains were transferred to $2 \%$ dimethyl sulfoxide (DMSO; VWR, USA) solution for cryoprotection for 1-2 days, after which the Neuropixel shanks were removed, the brains were frozen and sectioned in 50um slices with a sliding microtome (Microm HM430, Thermo Fisher Scientific, USA). The tissue sections then underwent fluorescent immunostaining against NeuN (catalog no.ABN90P, Sigma-Alrich, USA), followed by secondary antibody-staining with Alexa 647-tagged goat anti-guinea pig antibody (catalog no.A21450, Thermo Fisher Scientific, USA), after which the sections were rinsed, mounted, coverslipped and stored at $4^{0} \mathrm{C}$. The detailed immunostaining protocol is available per request. Next, the sections were digitized using an automated scanner for fluorescence and brightfield images at the appropriate illumination wavelengths (Zeiss Axio Scan.Z1, Jena, Germany), and were saved in .jpeg format for processing in TRACER.

Magnetic Resonance Imaging: MRI scanning was performed on a $7 \mathrm{~T}$ MRI with a $200 \mathrm{~mm}$ bore size (Biospec 70/20 Avance III, Bruker Biospin MRI, Ettlingen, Germany); an 86mm diameter volume resonator was used for RF transmission, and a phased array rat head surface coil was used for reception. Brains were submerged in fluorinert (FC-77, 3M, USA) to remove background signal on MRI. A 3D T1 weighted FLASH sequence was acquired at $0.06 \mathrm{~mm} 3$ resolution (TE: $10 \mathrm{~ms}$, TR: $40 \mathrm{~ms}$, NA: 4, matrix size: $360 \times 256$ x 180, FOV: $21.6 \mathrm{~mm}$ x $15.4 \mathrm{~mm}$ x $10.8 \mathrm{~mm}$, acquisition time: 2 hours 20 minutes).

Virus expression: pulled glass pipettes were used to inject $500 \mathrm{~nL}$ of AAV5-mDIx-Chr2-mCherry-Fishell-3 (plasmid no.83898, Addgene, USA; AAV produced at Kavli Viral Vector Core Facility, NTNU) at a rate of $50 \mathrm{~nL}$ per minute. Bilateral injections were targeted to the cingulate cortex $(\mathrm{Cg} 1 ;+2.0 \mathrm{AP}, \pm 0.5 \mathrm{ML},-2.0 \mathrm{DV}) .5$ weeks after surgery the animal was sacrificed and perfused with $4 \%$ paraformaldehyde. The brain was removed, post-fixed overnight in $4 \%$ paraformaldehyde at $4^{0} \mathrm{C}$, then cryoprotected for $24 \mathrm{hr}$ in $2 \%$ DMSO. The brain was then frozen in dry ice and 40um sections were collected as described above. Fluorescent signal from the virus was amplified by immunostaining against Red Fluorescent Protein (RFP) (catalog no.5F8,

Chromotek GmbH, Germany), followed by secondary antibody-staining with Alexa 546-tagged Goat Anti-rat IgG (catalog no. A-11081, Thermo Fisher Scientific, USA). The detailed immunostaining protocol is available upon request.

\section{Results}

\section{General Outline}

TRACER was designed to serve as an intuitive, generic software tool that conducts three main functionalities: (i) planning the insertion of electrodes before surgery, (ii) locating and re-tracing electrode tracks from histological images, and (iii) visualizing the spread of virus or anatomical tracers. TRACER provides $3 \mathrm{D}$ visualisation windows for each of these main functionalities. Users can choose the functionality of interest by calling the corresponding scripts and performing a series of step-by-step actions using a mouse and keyboard.

Figure 1 shows the general structure of TRACER and the corresponding functions used in each of the three primary functionalities. The sequence of steps taken in the processing pipelines are shown with pictorial representations in Figure 2. Since TRACER uses the WHS atlas of the Sprague 
Dawley Rat Brain (Papp et al. 2014) as its anatomical reference, several data files from the WHS atlas are required to run TRACER, and are listed in the user manual on GitHub. Users can also download the corresponding files from the NITRC Waxholm Space atlas webpage and store all files in a folder on the local desktop. After TRACER has been installed on the local computer, the atlas can be loaded using the function:

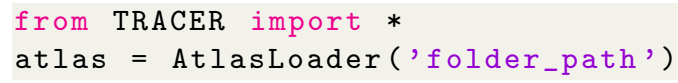

where 'folder_path' is the path of the folder where uses saved the atlas files.

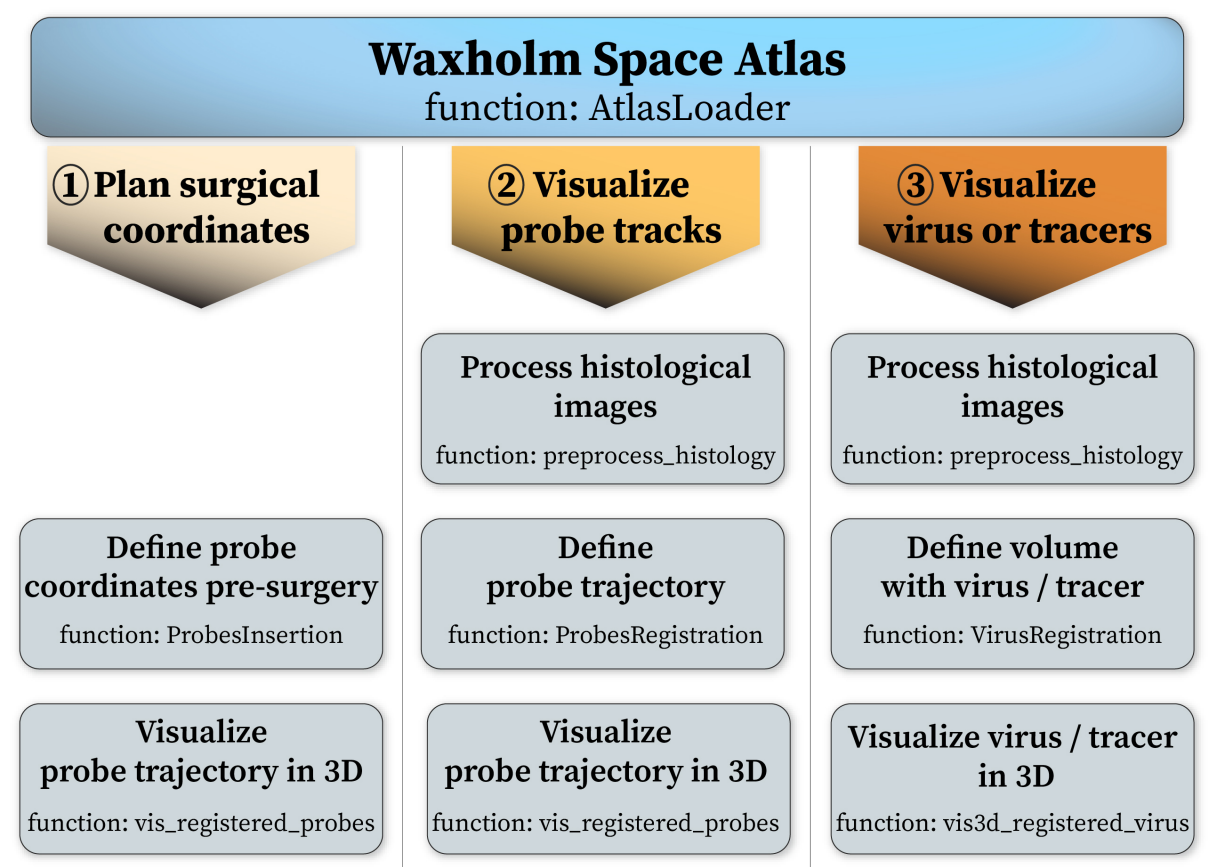

Figure 1. Flowchart showing the functions called in each of the major functionalities of TRACER. (1) Planning coordinates prior to surgery is done using the function ProbesInsertion, which can be visualised in 3D using the function vis_registered_probes. (2) Reconstruction of recording probes is achieved by processing histological images with the function: preprocess_histology, then recreating the trajectory with ProbesRegistration, which can be visualized in 3D with vis_registered_probes. (3) Viusalization of viral expression or anatomical tracers is done following the same steps as (2), but uses VirusRegistration for marking the boundaries of the ROI, and vis3d_registered_virus for visualizing the marked brain volume. 


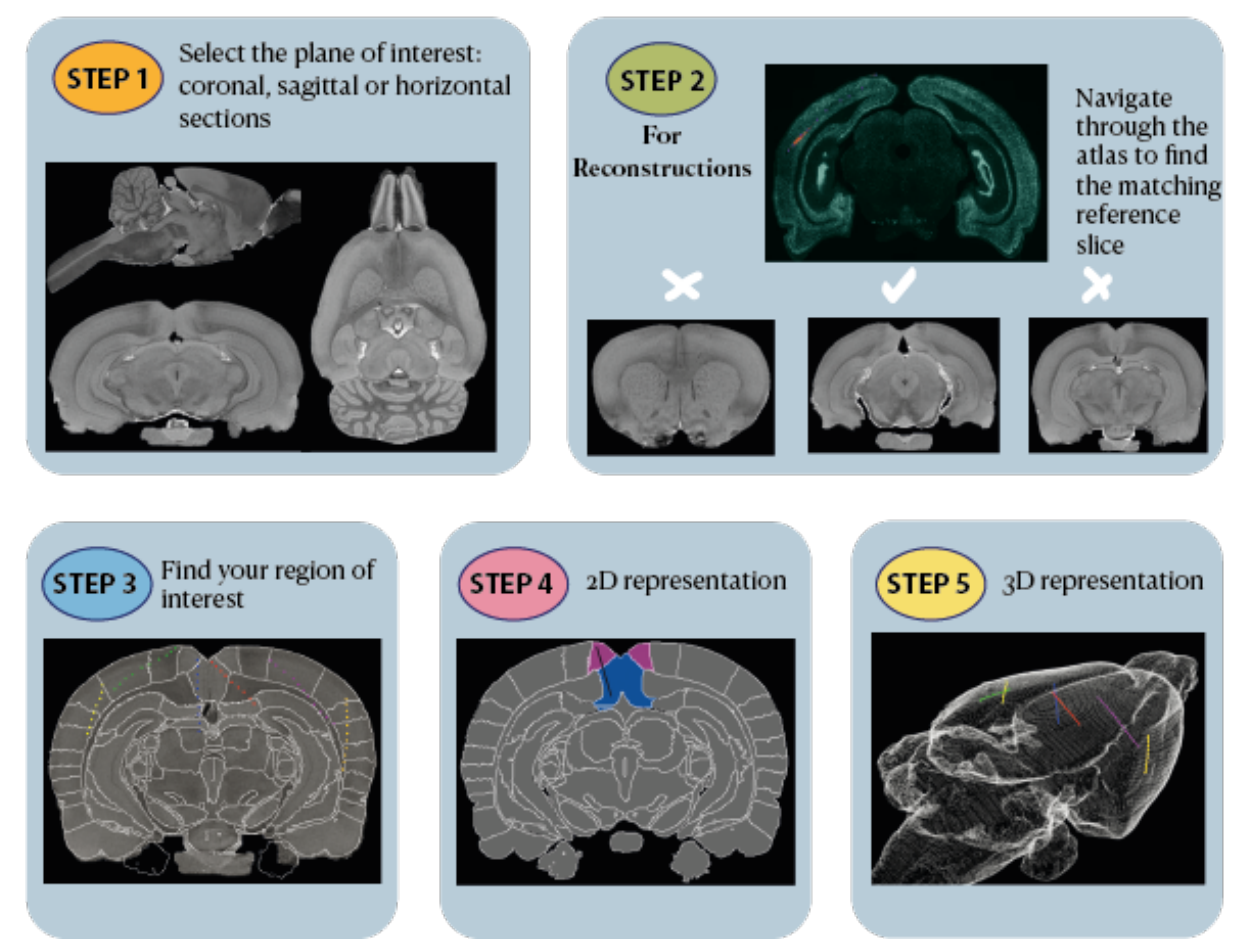

Figure 2. Schematic showing five major steps used in the TRACER processing pipeline. Step $\mathbf{1}$ is the selection of the plane for visualization (coronal, sagittal, horizontal). Step $\mathbf{2}$ applies only to post-hoc probe or anatomical reconstructions, and is to scroll through the Waxholm atlas brain sections to find the slice that best matches the user's histological section or contains the ROI. Step 3, after finding the ROI the user can either plan a probe insertion or mark points along the track of a previously-implanted probe; delineations from the WHS atlas appear at this stage. Step 4 provides a $2 \mathrm{D}$ visualization of the points highlighted in the previous steps; the example section shows a probe targeting retrosplenial cortex. Relevant information such as Bregma coordinates, insertion angle, probe length and regions traversed will be provided in a table when running the software. Step 5 produces a 3D rendering of the brain showing the location of recording probes or viral expression as defined in previous steps.

\section{Generating pre-surgerical coordinates}

One of the essential steps in planning a surgery is defining the stereotactic coordinates to reach specific brain areas of interest. TRACER significantly facilitates this process by giving the user a means to calculate the insertion parameters to target potentially several regions along a linear trajectory. This is done via an intuitive procedure of clicking points along the desired path on a $2 \mathrm{D}$ brain slice image. Once the trajectory is defined by the user, TRACER automatically computes the Bregma coordinates, insertion angle and length needed to reach the targets, which the user can print and bring to the surgery room or save for record keeping. Note that this functionality is equally applicable for targeting imaging lenses, injection pipettes or for planning focal lesions.

To illustrate the working steps of this functionality, we will plan here the insertion of a single-shank Neuropixels probe targeting visual cortex and the intermediate hippocampus. After calling the corresponding function shown in Figure 1, the annotated Waxholm atlas window appears,

obj = ProbesInsertion (atlas, 'probe_folder')

where probe_folder is the path of the directory in which the inserted probe's data will be be saved, or the path of the directory that contains the inserted probe's data to be loaded.

The user first scrolls to the brain slice of interest (Figure 3A), then defines the probe trajectory 

available under aCC-BY-NC-ND 4.0 International license.

through target regions with clicks of the mouse (Figure 3B). Once the probe trajectory is completed and saved, a 2D representation is generated showing the trajectory of the probe in a Waxholm brain slice (Figures 3C and 3D) along with a table including the location, inclination, length of probe to be inserted, regions traversed, and number of recording channels per region; this can saved automatically in a folder inside TRACER. Furthermore, it can be visualized in 3D (Supplementary material: Video 1). At the moment TRACER allows users to plan the insertion of up to 6 probes at a time, which is done by simply repeating the steps above for each probe.
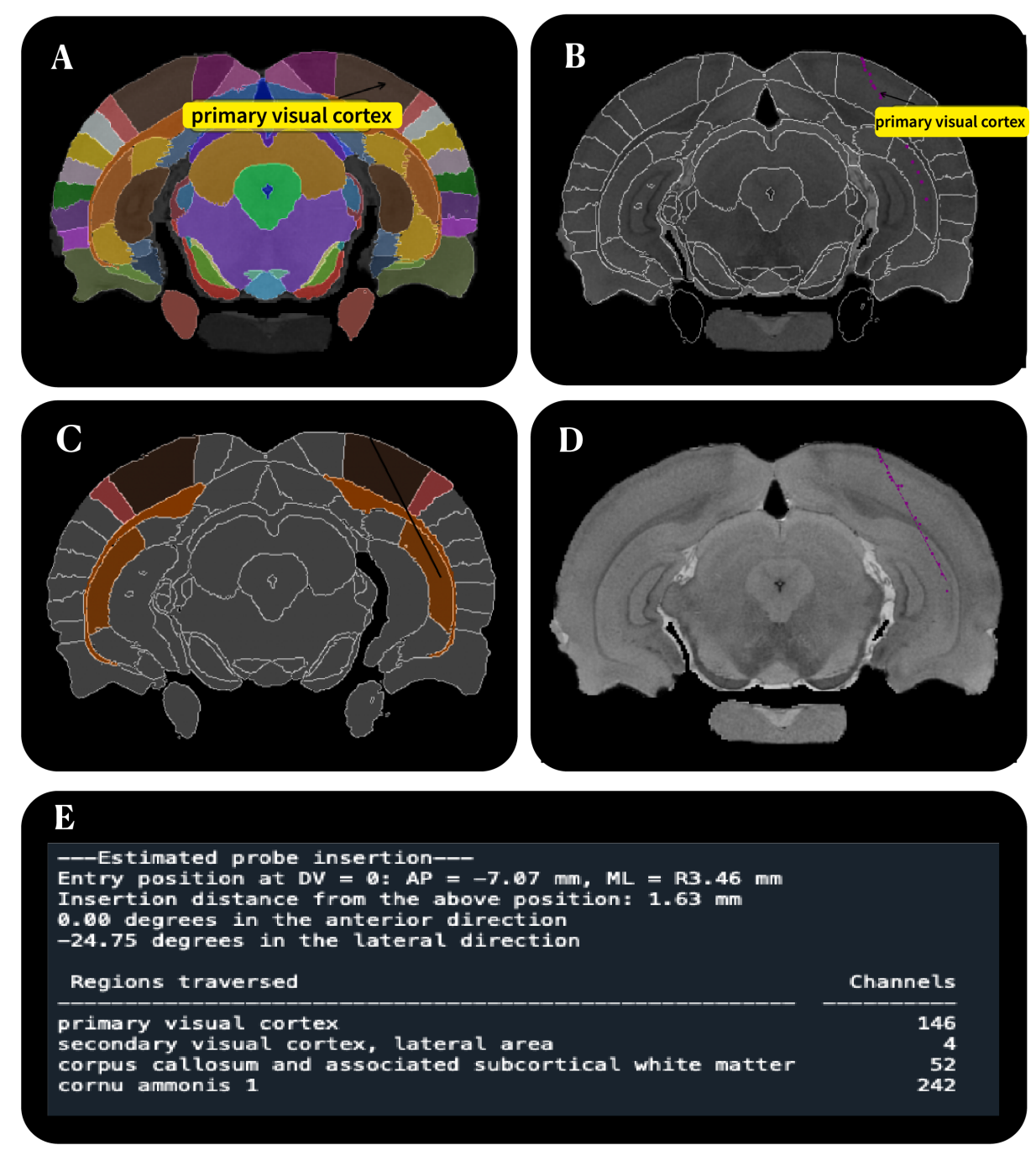

Figure 3. Using TRACER to plan a Neuropixels probe implant before surgery. (A) Once the plane of choice is set (coronal in this example), 2D brain sections with regional delineations appear and the user scrolls to the slice with the region(s) they wish to target. (B) The user clicks along the desired trajectory of the probe; regional boundaries remain outlined to facilitate accuracy. (C) TRACER uses the clicked points from $\mathbf{B}$ to generate an output which highlights in color the regions the probe will contact. (D) A linear probe trajectory is projected into the Waxholm space in 2D. (E) A text readout provides the entry position, probe angle and insertion length to reach the target regions, as well as an estimation of the number of recording channels in each region in the ensuing experiments. 


\section{Analysis and retracing of probe trajectories}

In order to reconstruct probe trajectories after surgeries or in vivo recordings, histological images must be pre-processed before they are overlaid on their corresponding atlas images. Histological images can be adjusted in TRACER by calling the following function,

preprocess_histology ('histology_folder')

where 'histology_folder' is the path of the directory in which the histological images are stored. Once this function is executed the console requests the name of the histology image. After the image is loaded and has been adjusted, the user calls the function ProbesRegistration. The working steps of the reconstruction pipeline are shown in (Figure 4), which uses an example section with the track from a Dil-stained Neuropixels probe (red) extending from visual cortex into the intermediate hippocampus.

The first step is to choose the plane of sectioning (coronal, sagittal, horizontal) and to navigate to the corresponding Waxholm atlas brain slice. Once the corresponding slice is located, the user defines anchor points to fit the histological section onto the atlas section (Figure 4A). The user then clicks where the probe track is visible in the tissue (Figure 4B), after which a 2D rendering is generated showing the probe and the brain regions it contacts (Figure $4 \mathrm{C}$ ), and a table showing the inclination and length of the probe, regions traversed and number of recording channels per region. This information is stored automatically in a sub-directory with name 'Probes' within the given histology folder. Regardless of the plane of sectioning, we found the best results required a minimum of 4 clicked points along the length of a $6 \mathrm{~mm}$ Neuropixels probe track. Neuropixels users should note that estimates of probe length include the 175um channel-less shank tip, and that TRACER eliminates it when estimating the number recording channels in the brain. 

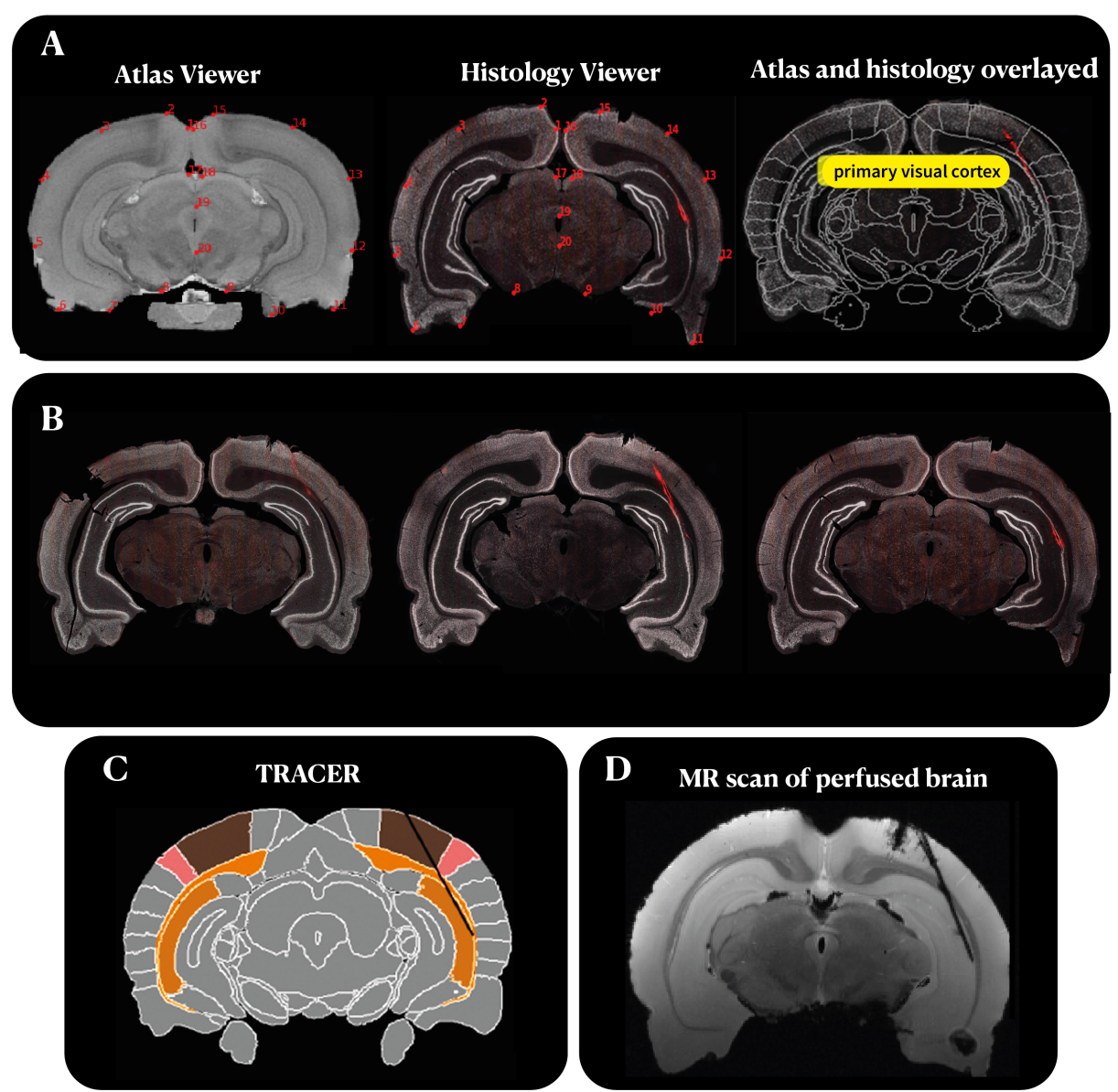

Figure 4. Reconstructing a recording probe trajectory from histological sections. (A) The initial steps include scrolling to the atlas brain slice (left panel) that best matches the user's histological section (middle panel). User-defined anchor points are then used to stretch and conform the histological section onto the atlas section; once they are merged regional delineations from the Waxholm atlas appear automatically (right panel). (B) The user then generates an estimate of probe placement and length by clicking at least four points spanning from the entry of the probe in the brain to the distal tip; the Neuropixel track in the example spans from visual cortex to hippocampus in three consecutive sections. (C) Shows the estimated probe placement following the steps in $\mathbf{A}$ and $\mathbf{B}$, with brain regions contacted by the probe highlighted in color. (D) A coronal section from an MRI scan of the original brain with the probe inside confirms the similarity between "ground truth" and TRACER output.

We assessed the accuracy of the reconstructions by comparing TRACER output against MRI scans taken of the same brains while they were intact, which appeared to show a strong correspondence for probe placement, length and regions traversed (Figure $4 \mathrm{C}$ and $\mathrm{D}$ ). To quantify these observations we made further comparisons in six additional brains, three of which had Neuropixels probes implanted in the coronal plane and three in the sagittal plane. We found that measures of probe length from the MRI scans and from TRACER yielded a strong goodness-of-fit across methods $\left(\mathrm{R}^{2}=0.93\right.$; mean error of $0.26 \mathrm{~mm}$, or $4.2 \%$ of "ground truth" from MRI scans; Figure 5B). In addition, we compared estimates of the number of recording channels in the brain made either by locating the channel at which the low-band LFP shifted as the probe exited the brain (Siegl et al. 2019b) or by anatomical reconstruction with TRACER. We again found a strong goodness-of-fit between methods $\left(\mathrm{R}^{2}=0.77\right.$; mean error of 17.1 out of 632.7 channels, $2.7 \%$ of 
"ground truth" per probe; Figure5A). We noted that the strength of this correlation was not as strong as that for probe length estimates, which could be attributable to the fact that the animals were still alive during the LFP-based channel counts, whereas TRACER used tissue after the brains had been fixed with paraformaldehyde, which causes non-uniform tissue shrinkage. The accuracy of channel counts and probe length estimates did not differ whether the probes were implanted parallel or tangential to the plane of the sectioning.

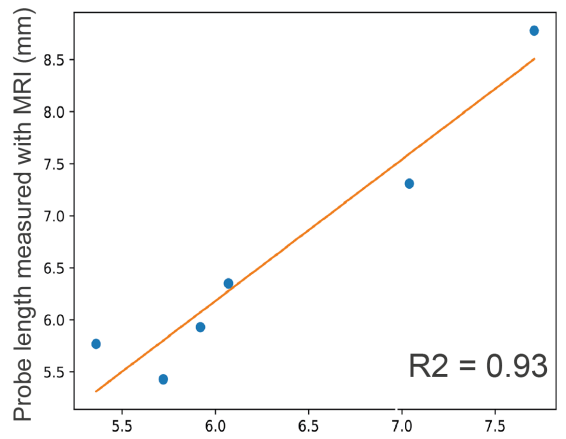

Probe length estimated by TRACER $(\mathrm{mm})$

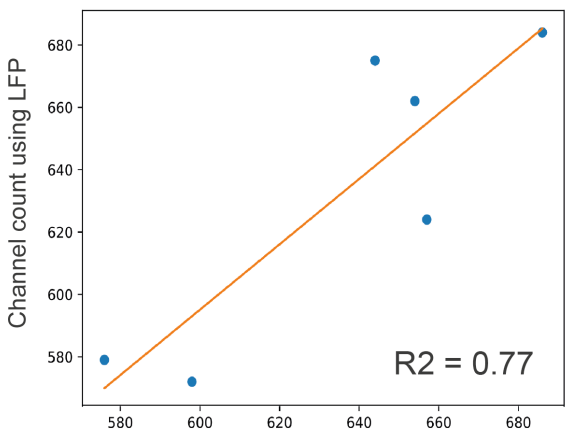

Channel count estimated by TRACER

Figure 5. Comparison of TRACER output against "ground truth" measurements. (Left panel) Analysis of six rat brains with chronic Neuropixels implants showed a strong goodness-of-fit between probe length measurements made from MRI scans versus reconstructions generated by TRACER. (Right panel) The same was true for estimates of the number of recording sites in the brain, which were calculated either using in vivo LFP measurements or TRACER.

\section{Visualization of virus expression}

TRACER can also be used for anatomical applications such as visualization of virus expression, anatomical tracer labeling, or demarcating lesions or neurodegeneration. Here we provide a brief overview of the procedure for reconstructing a spatial volume of adenovirus (AAV) expression. The procedure is similar to that for probe reconstruction, except the regions defined in each slice are 2-dimensional and the volume to be delineated extends over multiple slices (Figure 6). As with reconstructing a probe track, the first step is pre-processing hisotlogical images using the function preprocess_histology (Figure 1), followed by locating the correct starting slice in the atlas and overlaying the relevant sections. The user then defines the perimeter of virus expression by clicking around the border of fluorescence in the tissue (Figure 6B); these steps are repeated for each slice the user wishes to include in the visualization. Finally, using vis3D_registered_virus, the marked regions from several 2D sections are combined and projected in a 3D brain (Figure 6C and Video 2) with regional labels and coordinates outlining viral expression. The same functions and methods can be used for related tasks such as labeling single cells, marking fibers of passage or visualizing lesions. Note that the granularity of the reconstruction depends on how many points the user clicks per slice and how many sections the user includes in the reconstruction. The example in Figure 6C comprised 21 slices of histology with approximately 30 clicked points per slice. 


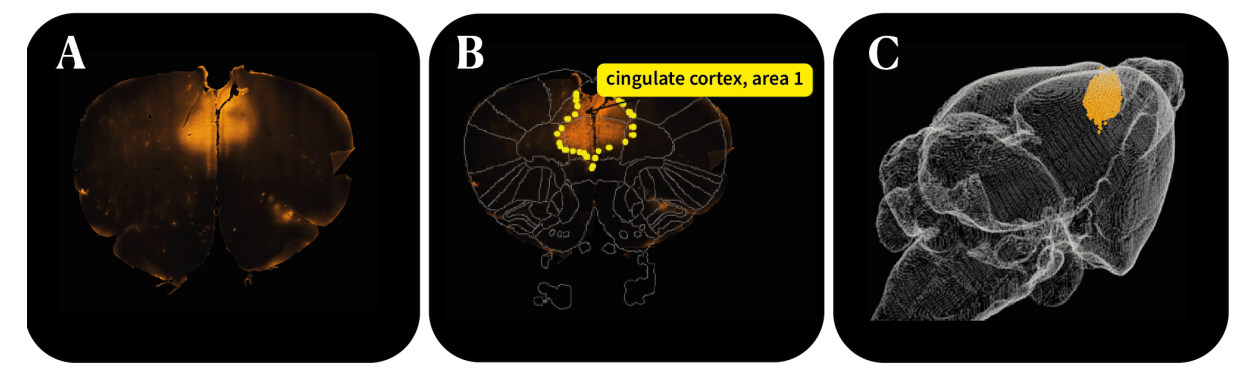

Figure 6. 3D visualization of virus expression across multiple tissue sections. (A) Shows the spatial extent of AAV-mediated mCherry expression in a coronal section from the frontal cortex of an adult rat. (B) The region of viral expression is defined by clicking points around the perimeter of expression; areal boundaries are included at this stage. (C) A volumetric rendering of the brain created using Vedo shows the extent of viral expression in the cingulate cortex.

\section{Discussion}

With TRACER we seek to provide rat users with a beginner-friendly python interface to facilitate accurate surgical implantations as well as the registration and visualization of anatomical data in a precise, curated 3D volume. The software works with potentially any histological staining method as long as the input images can be matched with corresponding template slices in the WHS rat brain atlas. Since template matching is done for each slice individually, and since the user marks regions of interest on images of the tissue directly, this framework should greatly reduce subjective judgement and variability in reconstructing electrode tracks or other anatomical features. TRACER also references the newest version (v4) of the WHS rat brain atlas (Papp et al. 2014), an open source, comprehensively delineated template containing over 200 labeled cortical and sub-cortical brain regions at sub-millimeter scales.

\section{Sources of error and future improvements}

While we hope that TRACER proves useful in its current form, there are areas where it could be improved in the future. For example, one potential source of error is that the software currently assumes a purely coronal, saggital and horizontal plane of sectioning, but often times tissue is sectioned slightly out of plane or unevenly across hemispheres. To deal with this, future versions could allow the user to rotate the Waxholm brain template to better match the cutting plane of tissue series individually. In addition, pre-processing and template matching steps in the present version can be time-consuming and repetitive if data sets contain a large number of sections, as with virus expression or tracer labeling. We aim to streamline these processes by implementing algorithms for pre-processing user images and matching reference slices in subsequent versions. Lastly, though we designed TRACER to require minimal or no programming skill, it can nevertheless be challenging for users who have not installed python and anaconda previously. We are therefore working to make TRACER accessible as a standalone, click-button graphical user interface (GUI) for download in the near future.

In developing TRACER we also encountered sources of error which appear general to the process of reconstructing anatomical volumes programmatically using fixed tissue. Specifically, we found that probe length estimates became less consistent as the number of histological slices used for reconstructions increased. We suspect that these errors arise from averaging across clicked points to define the trajectory the probe trace, and it is possible that misalignment of clicked points between slices adds to this error. Another issue noted in the Results was that the process of fixing the tissue with paraformaldehyde causes non-uniform shrinking of the tissue. This could account for the weaker goodness of fit when comparing channel counts from TRACER, performed after animals were perfused, against LFP measures taken while the animals were still alive. The probe length estimates 
from MRI scans and TRACER, on the other hand, both used fixed tissue and were more strongly correlated. Since there is no definitive solution for these sources of variability we recommend that users perform reconstructions more than once and use the most moderate estimates for their records.

Accurately registering raw neuroanatomical data in a reference template, though important, is usually tedious and time consuming. TRACER was developed to expedite, simplify and systematize this process by furnishing users with anatomical coordinates, annotated 2D and 3D visualizations and data tables summarizing its outputs. As a registration tool for the rat brain it complements existing varieties of software for rendering and registering anatomical coordinates in mice and other species. We wish to emphasize that TRACER is a completely open source software with which we aim to help our fellow research community. We therefore welcome users to submit suggestions for improvements and to report bugs on our GitHub page, as this will help us continually improve the software and user experience.

\section{Data availability, software and citation policy}

The software described in this manuscript is an open-source software written completely in python 3.8. TRACER is fully supported by Windows, macOS and Linux. Source code is available on https://github.com/Whitlock-Group/TRACER. User manual and documents are available on the Whitlock group Github page.

The Waxholm Space rat brain atlas files can be downloaded from the NITRC website (https://www.nitrc.org/projects/whs-sd-atlas). The required atlas files to run TRACER can be downloaded using the links below and cited as follows:

WHS rat brain atlas $\mathrm{v} 4$ :

- WHS_SD_rat_atlas_v4.nii.gz (Kleven et al., in preparation)

- WHS_SD_rat_atlas_v4.label (Kleven et al., in preparation)

Previous version of the WHS rat brain atlas (v1.01):

- WHS_SD_rat_T2star_v1.01.nii.gz (Papp et al. 2014)

- WHS_SD_rat_brainmask_v1.01.nii.gz (Papp et al. 2014)

Citation Policy:

We kindly ask users to cite this paper when using TRACER in their studies, and to cite the appropriate version of the Waxholm Space rat brain atlas.

Refer to the WHS atlas by its RRID: SCR_017124, and cite the first publication (Papp et al. 2014) along with the version of the atlas that is used. For example, cite Kjonigsen et al. 2015 , v2 of the atlas if the user's work makes particular use of delineations of the hippocampal region. Or cite Osen et al. 2019, v3 of the atlas if the user's work makes particular use of delineations of the auditory system.

License:

The Waxholm Space atlas of the Sprague Dawley rat brain is licensed under the Creative Commons Attribution ShareAlike (CC BY-SA) 4.0 license:

https://creativecommons.org/licenses/by-sa/4.0/

\section{Acknowledgments}

We thank T. B. Leergaard, I. E. Bjerke and H. Kleven for graciously sharing and assisting with the Waxholm Space atlas version 4; T. Tombaz, B. Mimica, E. H. Holmberg and I. Rautio for sample tissue; M. Andresen for technical assistance; D. Hill and M. Widerøe of the MRI Core Facility, Norwegian University of Science and Technology (NTNU); E. H. Holmberg, B. Kanter, T. Tomaz and I. Rautio for beta testing TRACER functionalities; M. P. Witter and members of the Whitlock 
lab for helpful discussion; B. Mimica and T. Tombaz for motivating the need for such a toolkit. This work was supported by a Research Council of Norway FRIPRO grant (No. 300709) to J.R.W., the Centre of Excellence scheme of the Research Council of Norway (Centre for Neural Computation, grant No. 223262), the National Infrastructure scheme of the Research Council of Norway NORBRAIN (grant No. 197467), and The Kavli Foundation. The MRI core facility is funded by the Faculty of Medicine at NTNU and Central Norway Regional Health Authority.

\section{Author contributions}

Jacopo Paglia, Kavli Institute for Systems Neuroscience, NTNU, Trondheim, Norway. Present address: KnowBe4, Apotekergata 10, Oslo, Norway. Contribution: Software design and development, Validation, Visualization, Conceptualization. For correspondence: jacopo.paglia91@gmail.com. Competing interests: No competing interests declared.

Pearl Saldanha, Kavli Institute for Systems Neuroscience, NTNU, Trondheim, Norway. Contribution: Software design, Validation, Visualization, Writing - original draft, Writing - review and editing. For correspondence: pearl.saldanha@ntnu.no. Competing interests: No competing interests declared.

Jingyi Guo Fuglstad, Kavli Institute for Systems Neuroscience, NTNU, Trondheim, Norway. Contribution: Conceptualization, Supervision, Software design and development, Validation, Visualization. For correspondence: jingyi.guo@ntnu.no. Competing interests: No competing interests declared.

Jonathan R. Whitlock, Kavli Institute for Systems Neuroscience, NTNU, Trondheim, Norway. Contribution: Conceptualization, Supervision, Project administration, Funding acquisition, Writing original draft, Writing - review and editing. For correspondence: jonathan.whitlock@ntnu.no.

Competing interests: No competing interests declared.

\section{References}

[1] E. Calabrese et al. "A quantitative magnetic resonance histology atlas of postnatal rat brain development with regional estimates of growth and variability". In: Neuroimage 71 (2013), pp. 196-206.

[2] F. Claudi et al. "Visualizing anatomically registered data with brainrender". In: Elife 10 (2021), e65751.

[3] D. Fürth et al. "An interactive framework for whole-brain maps at cellular resolution". In: Nature neuroscience 21.1 (2018), pp. 139-149.

[4] K. B. F. George Paxinos. The Mouse Brain in Stereotaxic Coordinates, Compact 3rd Edition. 2008.

[5] C. R. Harris et al. "Array programming with NumPy". In: Nature 585 (2020), 357-362. DOI: $10.1038 / s 41586-020-2649-2$.

[6] T. Hjornevik et al. "Three-dimensional atlas system for mouse and rat brain imaging data". In: Frontiers in Neuroinformatics 1 (2007), p. 4.

[7] J. D. Hunter. "Matplotlib: A 2D Graphics Environment". In: Computing in Science Engineering 9.3 (2007), pp. 90-95. DOI: 10.1109/MCSE.2007.55.

[8] L. J. Kjonigsen et al. "Waxholm Space atlas of the rat brain hippocampal region: three-dimensional delineations based on magnetic resonance and diffusion tensor imaging". In: Neuroimage 108 (2015), pp. 441-449.

[9] E. S. Lein et al. "Genome-wide atlas of gene expression in the adult mouse brain". In: Nature 445.7124 (2007), pp. 168-176. 
[10] B. Mimica et al. "Efficient cortical coding of 3D posture in freely behaving rats". In: Science 362.6414 (2018), pp. 584-589. ISSN: 0036-8075. DOI: 10.1126/science. aau2013. eprint: https://science.sciencemag. org/content/362/6414/584.full.pdf. URL: https://science.sciencemag. org/content/362/6414/584.

[11] S. W. Oh et al. "A mesoscale connectome of the mouse brain". In: Nature 508.7495 (2014), pp. 207-214.

[12] C. Ortiz et al. "Molecular atlas of the adult mouse brain". In: Science Advances 6.26 (2020), eabb3446.

[13] K. K. Osen et al. "Waxholm Space atlas of the rat brain auditory system: Three-dimensional delineations based on structural and diffusion tensor magnetic resonance imaging". In: Neuroimage 199 (2019), pp. 38-56.

[14] E. A. Papp et al. "Addendum to "Waxholm Space atlas of the Sprague Dawley rat brain"[NeuroImage 97 (2014) 374-386](10.1016/j. neuroimage. 2014.04. 001)". In: NeuroImage 105 (2015), p. 561.

[15] E. A. Papp et al. "Waxholm Space atlas of the Sprague Dawley rat brain". In: Neuroimage 97 (2014), pp. 374-386.

[16] P. Shamash et al. "A tool for analyzing electrode tracks from slice histology". In: BioRxiv (2018), p. 447995.

[17] J. Siegl et al. "Allen Institute ecephysspikesorting". In: (2019b). URL: https://github.com/AllenInstitute/ecephysspikesortin.

[18] J. H. Song et al. "Precise mapping of single neurons by calibrated 3D reconstruction of brain slices reveals topographic projection in mouse visual cortex". In: Cell Reports 31.8 (2020), p. 107682.

[19] A. W. Toga et al. "A 3D digital map of rat brain". In: Brain research bulletin 38.1 (1995), pp. $77-85$.

[20] G. Van Rossum and F. L. Drake. Python 3 Reference Manual. Scotts Valley, CA: CreateSpace, 2009. ISBN: 1441412697.

[21] P. Virtanen et al. "SciPy 1.0: Fundamental Algorithms for Scientific Computing in Python". In: Nature Methods 17 (2020), pp. 261-272. DOI: 10.1038/s41592-019-0686-2.

[22] S. Van der Walt et al. "scikit-image: image processing in Python". In: PeerJ 2 (2014), e453.

[23] Q. Wang et al. "The Allen mouse brain common coordinate framework: a 3D reference atlas". In: Cell 181.4 (2020), pp. 936-953.

[24] J. Winnubst et al. "Reconstruction of 1,000 projection neurons reveals new cell types and organization of long-range connectivity in the mouse brain". In: Cell 179.1 (2019), pp. 268-281.

[25] B. Zingg et al. "Neural networks of the mouse neocortex". In: Cell 156.5 (2014), pp. 1096-1111. 


\section{Supplementary material}

\section{Video 1}

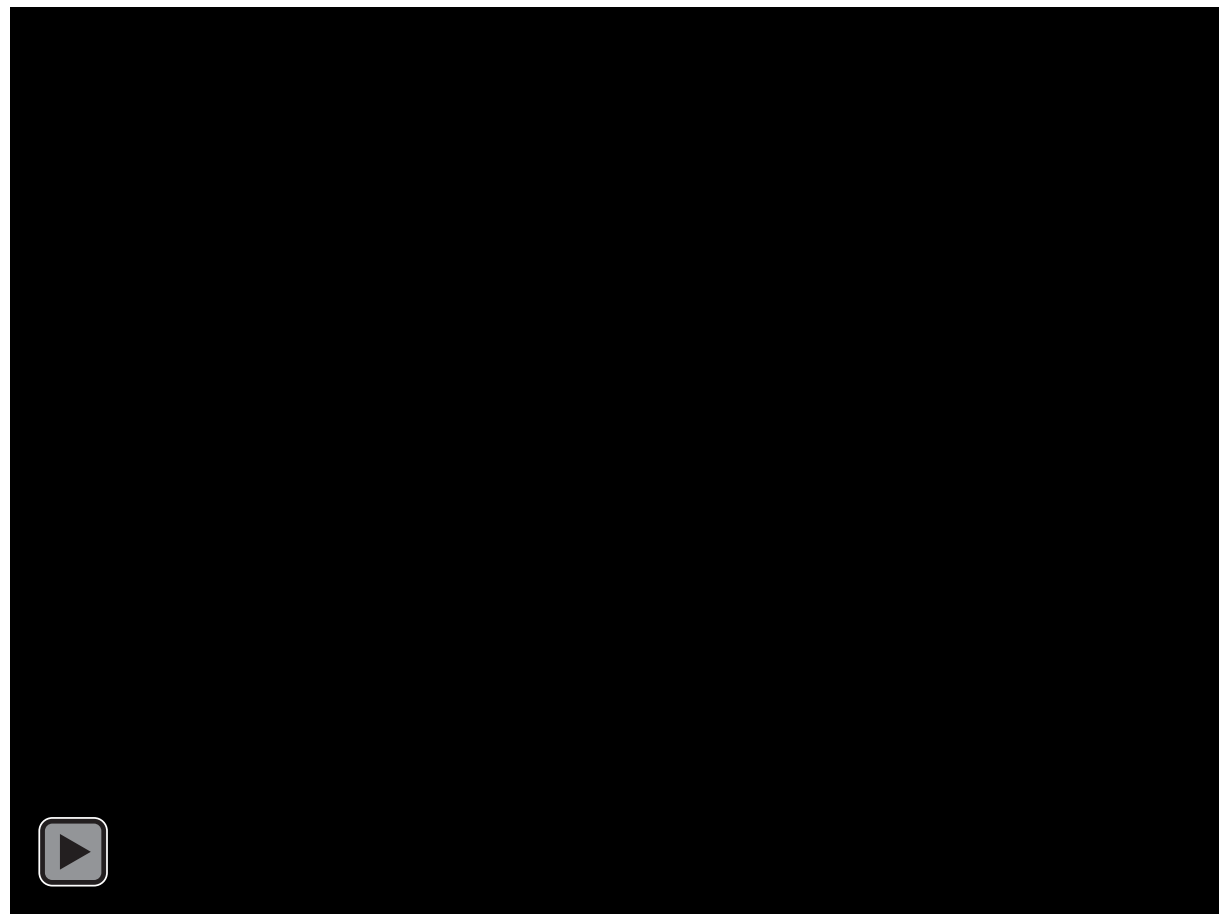

Video 1. 3D visualization of the inserted probe. The image can be rotated by left-clicking and dragging with the mouse; zoom can be adjusted using the mouse scroll wheel. 


\section{Video 2}

Video 2. 3D volumetric reconstruction of virus expression in the rat frontal cortex; controls are the same as in Video 1. 